\title{
Passive interferometric interrogation of a magnetic field sensor using an erbium doped fiber optic laser with magnetostrictive transducer
}

\author{
I.M. Nascimento ${ }^{\mathrm{a}, \mathrm{b}, *}$, J.M. Baptista ${ }^{\mathrm{a}, \mathrm{c}}$, P.A.S. Jorge ${ }^{\mathrm{a}, \mathrm{b}}$, J.L. Cruz ${ }^{\mathrm{d}}$, M.V. Andrés ${ }^{\mathrm{d}}$ \\ a INESC TEC, Rua do Campo Alegre, 687, 4169-007 Porto, Portugal \\ b Department of Physics and Astronomy, Faculty of Sciences of University of Porto, Rua do Campo Alegre 687, 4169-007 Porto, Portugal \\ ${ }^{\mathrm{c}}$ Centro de Competências de Ciências Exatas e de Engenharia, Universidade da Madeira, Funchal, Portugal \\ d Department of Applied Physics and Electromagnetism, University of Valencia, Spain
}

\section{A R T I C L E I N F O}

Article history:

Received 4 March 2015

Received in revised form 20 August 2015

Accepted 8 October 2015

Available online 22 October 2015

\section{Keywords:}

Magnetic field sensor

Passive interferometer

Virtual instrumentation

Magnetostrictive material

Erbium doped fiber

Fiber optic laser

\begin{abstract}
A B S T R A C T
An erbium doped $\left(\mathrm{Er}^{3+}\right)$ fiber optic laser is proposed for magnetic field measurement. A pair of FBGs glued onto a magnetostrictive material (Terfenol-D rod) modulates the laser wavelength operation when subject to a static or a time dependent magnetic field. A passive interferometer is employed to measure the laser wavelength changes due to the applied magnetic field. A data acquisition hardware and a LabVIEW software measure three phase-shifted signals at the output coupler of the interferometer and process them using two distinct demodulation algorithms. Results show that sensitivity to varying magnetic fields can be tuned by introducing a biasing magnetic field. A maximum error of $0.79 \%$ was found, for magnetic fields higher than $2.26 \mathrm{mT}_{\mathrm{RMS}}$.
\end{abstract}

(c) 2015 Elsevier B.V. All rights reserved.

\section{Introduction}

Fiber optic magnetic field sensors have been studied over the years. Special interest has been shown in the high power industry owing to its intrinsic insulation (silica), immunity to electromagnetic interference, high dynamic range and bandwidth and possibility to employ remote interrogation [1]. Several sensing mechanisms such as magnetic fluid, Lorentz force, Faraday effect and magnetostrictive effect have been proposed for magnetic field measurement. These methods can be used with both constant and varying magnetic fields.

The first mechanism was explored by combining the magnetic fluid with an optical fiber refractive index sensor. In the presence of the magnetic field the refractive index of the fluid changes $3 \times 10^{-4} \mathrm{RIU} / \mathrm{mT}$ (RIU-Refractive Index Units) at $28^{\circ} \mathrm{C}$ from 0 to $70 \mathrm{mT}[2]$.

Sensors established on the Lorentz force require another current carrying conductor (few milliamps) that will experience deformation in the presence of an orthogonal magnetic field. Although they do not experience hysteresis, the deformation induced is very

\footnotetext{
* Corresponding author.

E-mail address: ivomac88@gmail.com (I.M. Nascimento).
}

small; in [3] the optical sensor is a Distributed feedback laser, with a Pi-Shift FBG written in $\mathrm{Er}^{3+}$ fiber, and the wavelength changes are read with a Michelson interferometer with $25 \mathrm{~m}$ of optical path imbalance. A minimal detected field of $1.5 \mu \mathrm{T} / \mathrm{Hz}^{1 / 2}$ was calculated. Another alternative reported consists on using a $6 \mathrm{~cm}$ long cavity laser with one longitudinal mode and two orthogonal polarizations. Measurement of the beat frequency between these two polarizations is proportional to the laser birefringence and changes according to pressure exerted in the cavity due to the Lorentz force. Results showed relatively good linearity for magnetic fields between 4 and $20 \mathrm{mT}$ [4].

Faraday effect is one of the most popular optical sensing mechanisms for magnetic field. It consists of light polarization rotation induced by the magnetic field as it propagates through a sensing medium and its sensitivity depends on the medium Verdet constant. While standard fiber optic can be used as a sensing medium to observe the Faraday effect, the Verdet constant of silica is very small and the wounding of the fiber around the conductor gives rise to linear birefringence, further degrading the sensitivity [5]. A prototype operating at $850 \mathrm{~nm}$ and based on a Sagnac configuration was developed and tested where a maximum error of $0.2 \%$ was achieved for currents ranging from 300 to $4000 \mathrm{~A}_{\mathrm{RMS}}$ and temperatures from $40^{\circ} \mathrm{C}$ up to $60^{\circ} \mathrm{C}$, satisfying 0.5 class operation [6]. Faraday based sensors are affected by the residual birefringence of standard fibers [7], although this problem has been recently over- 


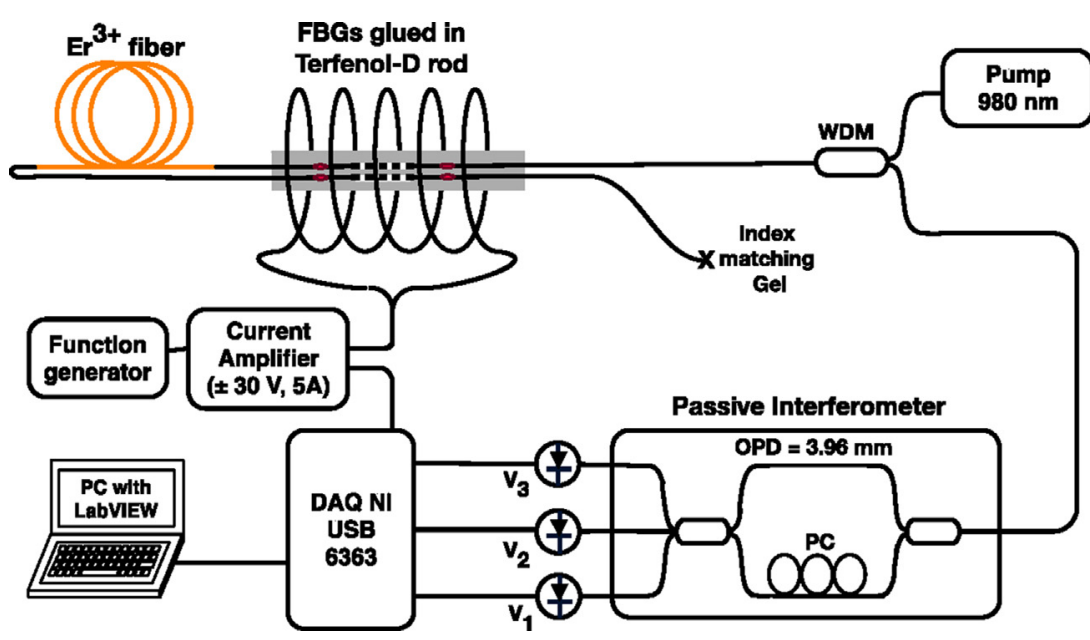

Fig. 1. Experimental setup including the laser, passive interferometer and acquisition setup.

comed in standard fibers [8], an alternative solution has been the use of spun fibers of low birefringence or high circular birefringence $[9,10]$. On the other hand, the bulk glass materials are more robust and can have higher Verdet constants than fibers. However, fiber alignment with the bulk material is tricky $[11,12]$. In [11] a sensor operating at $820 \mathrm{~nm}$ in a close loop configuration achieved a 5.5\% error under stable environmental conditions from $10 \mathrm{~A}-20 \mathrm{kA}$.

In the last category, magnetostricitve elements can be deposited or glued to an optical fiber strain sensor. In reference [13], TerfenolD is deposited in an etched FBG (Fiber Bragg Grating) with $86 \mu \mathrm{m}$ diameter, improving the sensitivity from 0.386 to $0.95 \mathrm{pm} / \mathrm{mT}$. A distributed sensor was also developed in [14] and consists in wounding a standard single mode fiber around a nickel wire. In the presence of the magnetic field the nickel wire stretches and the phase of the Rayleigh backscattered light changes according to it. A passive Mach-Zehnder interferometer with a $3 \times 3$ coupler and an OPD of $2 \mathrm{~m}$ ( $1 \mathrm{~m}$ spatial resolution) was used to read the phase changes.

In this paper we present an erbium doped optical fiber laser with two FBGs whose wavelength is modulated according to the external magnetic field. The transducer element is a Terfenol-D rod that stretches both FBGs, changing the laser emission wavelength. This variation is converted into an intensity modulation at the output of a passive interferometer using a $3 \times 3$ output coupler. The laser combines higher SNR (Signal to Noise Ratio) with narrower bandwidth, enabling, together with the interferometric readout system, a higher resolution than is attainable with systems based on the direct modulation of a single FBG.

\section{Principle}

The implemented setup is demonstrated in Fig. 1. The laser consists of two FBGs at $1534.17 \mathrm{~nm}$ with $150 \mathrm{pm}$ spectral width (at half power) and $82 \%$ reflectivity, and the other at $1534.21 \mathrm{~nm}$ with $160 \mathrm{pm}$ spectral width and $87 \%$ reflectivity, written in single mode Boron codoped Photosensitive fiber using a $1058 \mathrm{~nm}$ period phase mask. In between the two FBGs, a piece of $6.8 \mathrm{~m}$ of Fibercore Erbium doped fiber M-5 is used as the gain medium, resulting in $8 \mathrm{~m}$ cavity length.

Each FBG is glued side by side in two points, distant $2 \mathrm{~cm}$ apart, in a Terfenol-D (composition $\mathrm{Tb}_{0.27} \mathrm{Dy}_{0.73} \mathrm{Fe}_{2}$ ) rod having a diameter of $0.5 \mathrm{~cm}$ and $10 \mathrm{~cm}$ in length. The thickness of the material limits the magnetic field frequency response to $100 \mathrm{kHz}$. A function generator, a current amplifier and an inductor are used to generate magnetic field (AC and/or DC with a magnetic-current relation of $12.2 \mathrm{mT} / \mathrm{A}$ ), modulating both FBGs and consequently the laser wavelength emission. The AC and the DC magnetic field correspond to the alternate and constant field, respectively. Laser operation in reflection is preferable than in transmission as no residual pump power is present in the output.

For the detection of the magnetic field induced wavelength shift, an interferometric readout scheme was set up. A passive interferometer, having a OPD of $3.96 \mathrm{~mm}$ (Optical Path Difference) resulting in a spectral range of $594 \mathrm{pm}$ between interferometric fringes at $1534 \mathrm{~nm}$ was built with a $2 \times 2$ and a $3 \times 3$ coupler at the input and output, respectively, producing three outputs with 120 degrees phase difference between them, given by [15]:

$V_{n}=A_{i}+B \cdot \operatorname{Cos}\left[\varphi(t)+\varphi_{\mathrm{DC}}-(n-1)\right] \frac{2}{3} \pi$

where $n$ is the output 1,2 and $3, A_{i}$ is the DC component obtained when sweeping one period of the interferometer, $B$ is the visibility of the fringes which is maximized by a polarization controller $(\mathrm{PC}), \varnothing(t)$ and $\emptyset_{\mathrm{DC}}$ is the time varying and DC interferometer phase, respectively. In such configuration, any change in the laser emission wavelength results in a change of the interferometer optical output phase $\left(\varnothing(t)\right.$ and $\left.\emptyset_{\mathrm{DC}}\right)$ proportional to the OPD. This way, the interferometer acts like a wavelength-to-intensity modulator enabling to track the wavelength changes, induced by the magnetic field, very accurately with low cost instrumentation.

This interferometer has the advantage of not needing an active element to avoid total output fading. The relative phase of the three outputs and the signal processing can always retrieve the relevant output information, independently of the random drift of the interferometer. Nevertheless, the interferometer drift is mixed with the DC phase changes, also affecting the output intensity and limiting the application of this scheme to AC measurements. In any case, magnetic field measurements were performed in a temperaturecontrolled environment.

A 16 bits analogue-digital converter from NI (National Instruments) with $305 \mu \mathrm{V}$ resolution and $2 \mathrm{Mbps}$ bandwidth is used to read the three outputs of the interferometer and the applied current signal to the inductor. In this way, the use of virtual instrumentation becomes possible, making it straightforward to test and implement any signal processing algorithm, by simply adjusting the software, offering a much higher versatility and scalability. Therefore, to test the versatility of virtual instrumentation systems, a LabVIEW program was developed to process the interferometric signals, and used to test and implement, simultaneously, two distinctive demodulation methods. The first one (type I) is presented in Fig. 2 and consists on performing derivatives and an integration as depicted [15]. The output only contains the variant phase infor- 


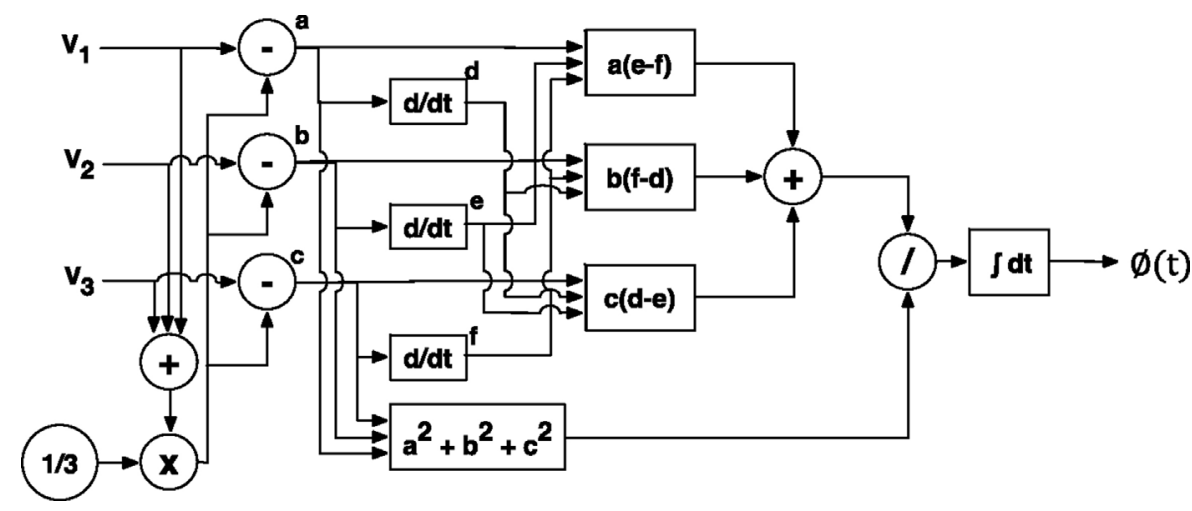

Fig. 2. Type I processing diagram.

mation and in order for this method to work properly the values of $A_{i}$ must be the same for the three outputs, which is achieved by introducing an adjustable gain in each interferometer signal.

The other implemented algorithm (type II) consists on performing the Arc tangent function of the signals provided by the detectors [16]:

$\varphi(t)+\varphi_{\mathrm{DC}}=\operatorname{ArcTan}\left[\frac{\sqrt{3}\left(a_{3} V_{2}-a_{2} V_{3}\right)}{a_{3} V_{2}+a_{2} V_{3}-2 a_{2} a_{3} V_{1}}\right]$

where $\alpha_{2}=A_{2} / A_{1}$ and $\alpha_{3}=A_{3} / A_{1}$. If the three outputs have the same gain then. $\alpha_{3}=1$ Although it is possible to recover the continuous phase information, the interferometer drift is also present and this method also requires an unwrapping algorithm to compensate phase changes out of $\pm p$.

Laser emission wavelength is temperature dependent and it is affected by the FBGs response to temperature and strain induced by the Terfenol-D rod due to thermal expansion. At the output of the interferometer this effect will be mixed with the random drift of the interferometer and using type I method this DC effects are excluded. On the other hand, when using the type II method the output will contain the temperature effect but having a slow variation which is simply removed by filtering the $\mathrm{AC}$ response.

\section{Results}

\subsection{Laser}

Laser spectral width is measured by coupling a tunable laser ( $100 \mathrm{kHz}$ bandwidth) with the developed one. In the frequency domain, the convolution of both incoherent signals is read with a $50 \mathrm{GHz}$ photodetector and an Electric Spectral Analyzer. Since the tunable laser is very narrow when compared to the developed laser the result gives the spectral shape of the laser, centered in the frequency given by the beat frequency of both [17]. In Fig. 3 it is shown the laser spectrum obtained with the electrical spectrum analyzer. A spectral width of $1.87 \mathrm{GHz}(14.7 \mathrm{pm}$ at $1534 \mathrm{~nm})$ was measured for a pump power of $560 \mathrm{~mW}$, the laser linewidth is 40 times smaller than the fringe spectrum range of the interrogation interferometer assuring adequate sensitivity. The linewidth dependence on pump power is residual and the variations are negligible with respect to the free spectral range of the interferometer, the slight benefits of the linewdtidth reduction are partially faded by the reduction of the emitted power, therefore, the pump level had little impact on the sensor performance. During the following measurements, the pump power was set at $560 \mathrm{~mW}$.

Using the Thorlabs PM20CH power meter the laser response was characterized using several pump power levels. Fig. 4 shows a maximum laser power of $4.7 \mathrm{~mW}$ for $560 \mathrm{~mW}$ pump and a threshold of $50 \mathrm{~mW}$ for lasing.

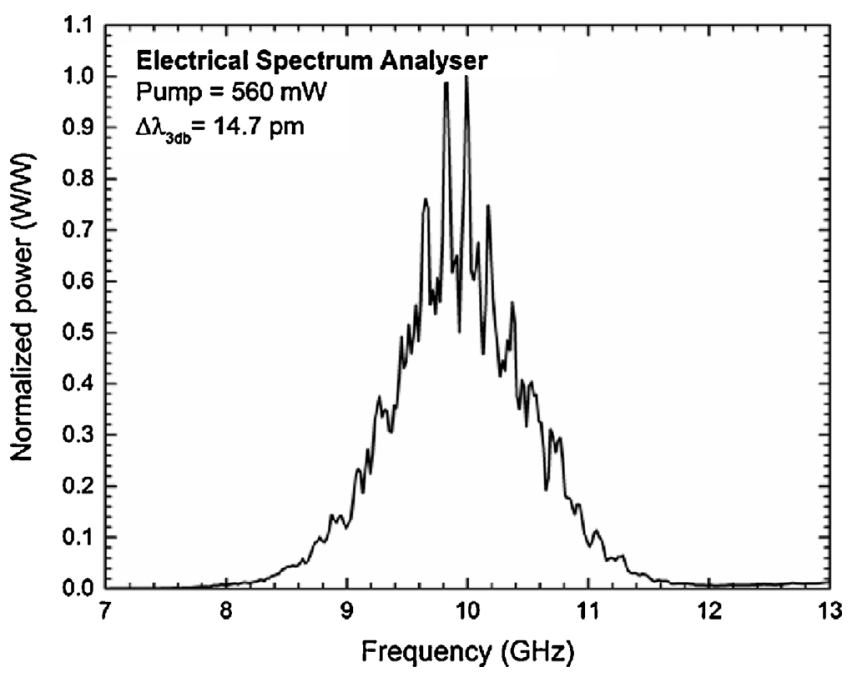

Fig. 3. Laser spectrum measured in an electrical spectral analyzer.

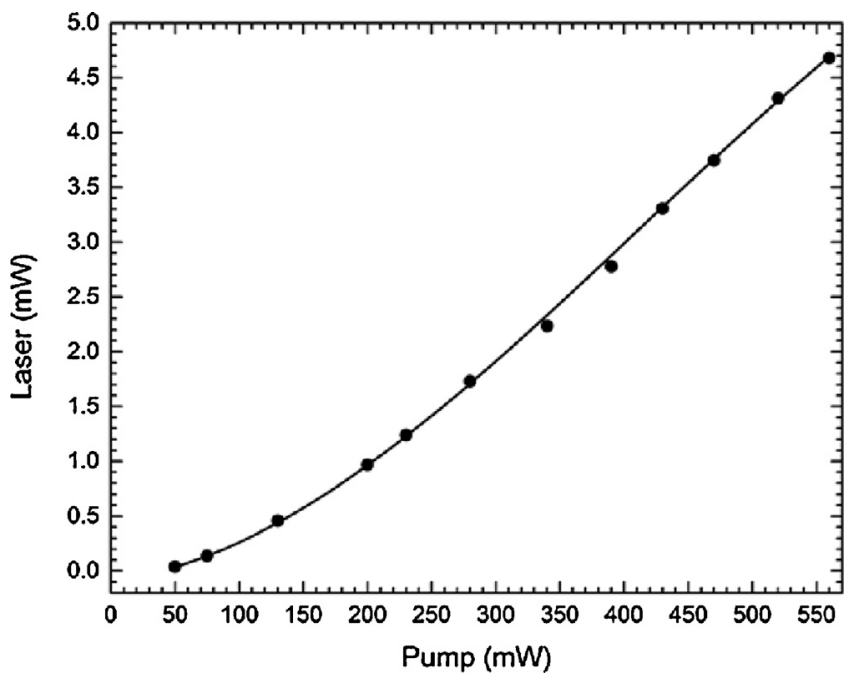

Fig. 4. Laser power in function of the pump power.

Laser power stability was also measured along the time using a photodetector and the analogue digital converter. Recording the laser power with an acquisition sampling frequency of $10 \mathrm{kHz}$ showed that the laser had an output power modulation below $1.2 \%$ at $50 \mathrm{~Hz}$ caused by the electronics driving the pump diode. Long term power fluctuations of about $4 \%$ where observed as well, however the detection electronics compensates for this slow variation. 


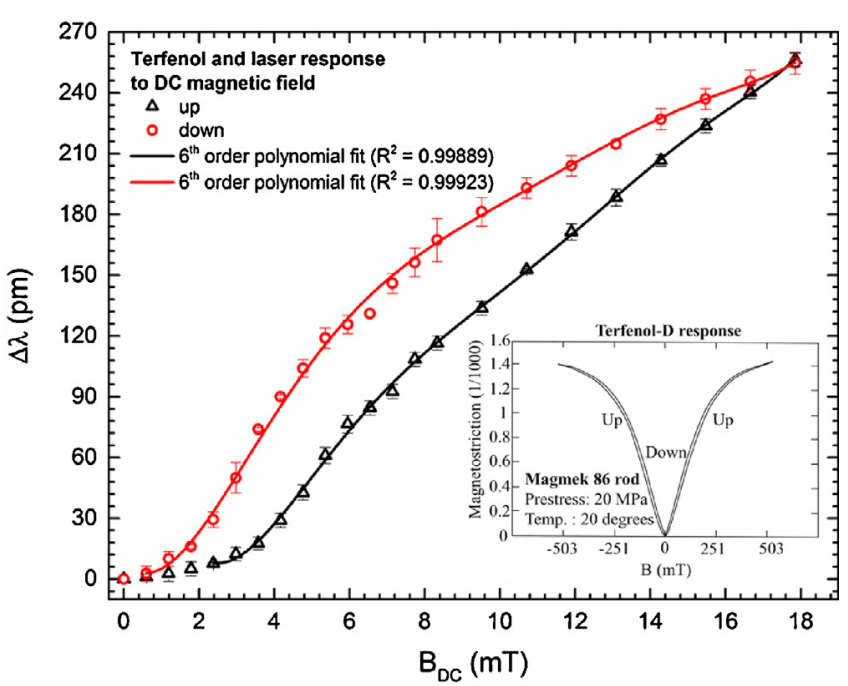

Fig. 5. Laser wavelength change with DC magnetic field and in the inset the material response according to the manufacture.

\subsection{Magnetic field measurement}

Several continuous current steps were applied to the coil and the laser wavelength changes were recorded using a wavelength meter Burleigh WA-1650 (0.5 pm resolution). Fig. 5 shows the magnetostrictive response of Terfenol-D driving the laser emission wavelength, the hysteresis cycle was obtained with three independent tests when the magnetic field goes up and down. The error bars correspond to the standard deviation of the three independent measurements, and account for the repeatability of the sensor, or the measurement precision. The maximum fluctuation registered was of $4.5 \mathrm{pm}$ at $B=5.95 \mathrm{mT}$ for the up curve and $10.7 \mathrm{pm}$ at $B=8.33 \mathrm{mT}$ for the down curve. In all cases, the measurement accuracy, given by the deviation between the measurement points and the non-linear calibration curve was much better, corresponding to smaller error values. The non-linear behavior of the calibration curve is intrinsic to the Terfenol-D response to DC magnetic fields, as can be seen in the inset of Fig. 5, where a representation of the manufacturer Data-sheet is given (notice that an unloaded rod was used, therefore saturation is reached with an applied field smaller than the one presented in the inset of Fig. 5). The material expansion in the presence of a magnetic field is non-linear and independent of the negative and positive sign of the magnetic fields. Therefore, when no bias magnetic field is applied, the application of an AC magnetic field results in a response that is doubled in frequency. Moreover, since the transducer is intrinsically non-linear, different DC biasing points will result in different sensitivities to AC fields. Hysteresis is an additional problem for DC measurements that is overcame with specific setups [18].

The laser response to alternate magnetic fields (AC) at $20 \mathrm{~Hz}$ was characterized using different constant magnetic fields (DC), with the setup presented in Fig. 1. The acquisition system was defined with $10 \mathrm{kHz}$ sample frequency and a low pass filter with $200 \mathrm{~Hz}$ cutoff was applied for each input signal, before processing the three outputs of the interferometer. The AC RMS value was obtained after filtering the demodulated signal with a second order Butterworth band-pass filter with a $5 \mathrm{~Hz}$ bandwidth.

Fig. 6 shows the RMS AC response for a DC magnetic field of $0 \mathrm{mT}$ applying different $\mathrm{AC}$ steps during $30 \mathrm{~s}$ each. The results were obtained with the demodulation process type II and the values shown in each step correspond to the average value. Response of the Terfenol-D to positive and negative magnetic fields is the same. So for $0 \mathrm{mT}$ magnetic field offset, the AC magnetic field pro-

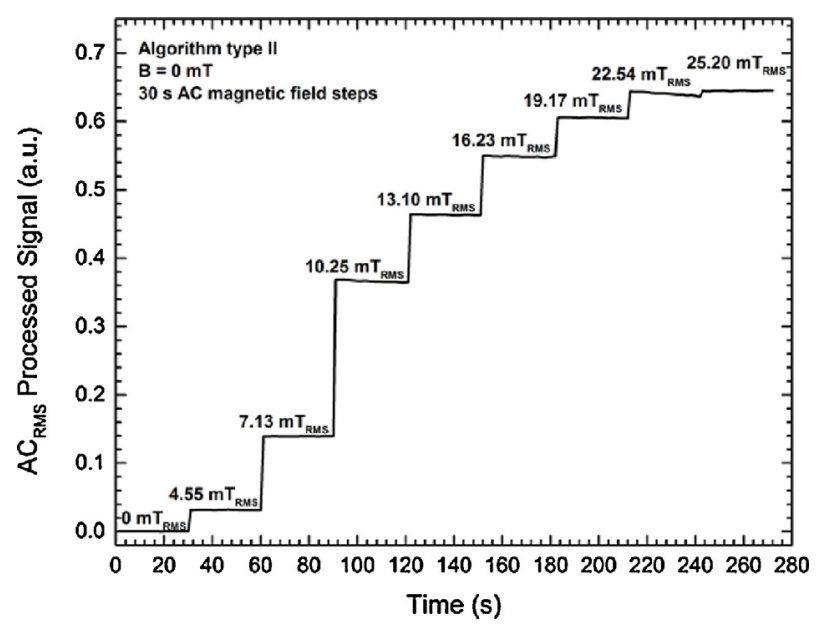

Fig. 6. Steps of $A C$ magnetic fields with $0 \mathrm{mT}$ offset.

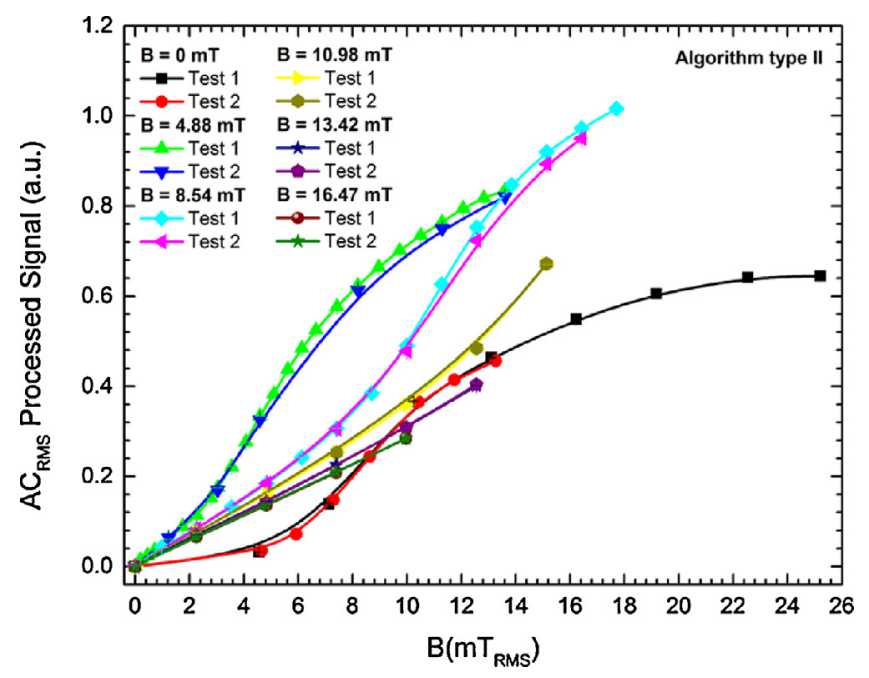

Fig. 7. Type II demodulation algorithm response to AC, using different magnetic field offsets.

duces a modulation whose frequency is acquired at two times the modulation frequency. Still when a magnetic field offset is introduced the modulation is therefore obtained at the same modulation frequency. Taking this effect into account, the acquisition will be acquired at to two times the modulation frequency when no bias magnetic field is present or at the modulation frequency when a bias field is applied. In Fig. 6 it is shown that for alternate magnetic fields below $4.55 \mathrm{mT}_{\mathrm{RMS}}$ the sensitivity is small, on the other hand, at values higher than $22.54 \mathrm{mT}_{\mathrm{RMS}}$ it saturates.

Compilation of the sensor response obtained using demodulation algorithm type II with different DC magnetic fields is present in Fig. 7. For each, two independent tests were conducted and the results showed good agreement. Also, a standard deviation error in each step is present, but they are too small to be observed in the plot. Analyzing these results we see that controlling the DC magnetic field offset (this could also be done by a permanent magnet) higher sensitivity can be obtained, depending of the measurement range. For magnetic fields up to $12.2 \mathrm{mT}_{\mathrm{RMS}}$ a constant field of $4.88 \mathrm{mT}$ gives the best response. On the other hand, for values higher than $12.2 \mathrm{mT}_{\mathrm{RMS}}$, a DC magnetic field of $8.54 \mathrm{mT}$ is preferable, measuring fields up to $18.2 \mathrm{mT}_{\mathrm{RMS}}$. Moreover the worst sensitivities were found for a constant magnetic field of $0,13.42$ and $16.47 \mathrm{mT}$, which also corresponded to the worst sensitivity region in Fig. 5. 
Table 1

Maximum AC detection errors in percentage obtained with different bias magnetic fields for variant magnetic fields higher than 2.25 mTRMS.

\begin{tabular}{|c|c|c|c|c|c|c|}
\hline Bias field B (mT) & 0.00 & 4.88 & 8.54 & 10.98 & 13.42 & 16.47 \\
\hline Type I (\%) & 1.609 & 0.406 & 0.645 & 0.776 & 0.655 & 0.792 \\
\hline Type II (\%) & 1.562 & 0.413 & 0.644 & 0.757 & 0.652 & 0.790 \\
\hline Relative Difference (\%) & 3.018 & -1.645 & 0.098 & 2.527 & 0.412 & 0.228 \\
\hline
\end{tabular}

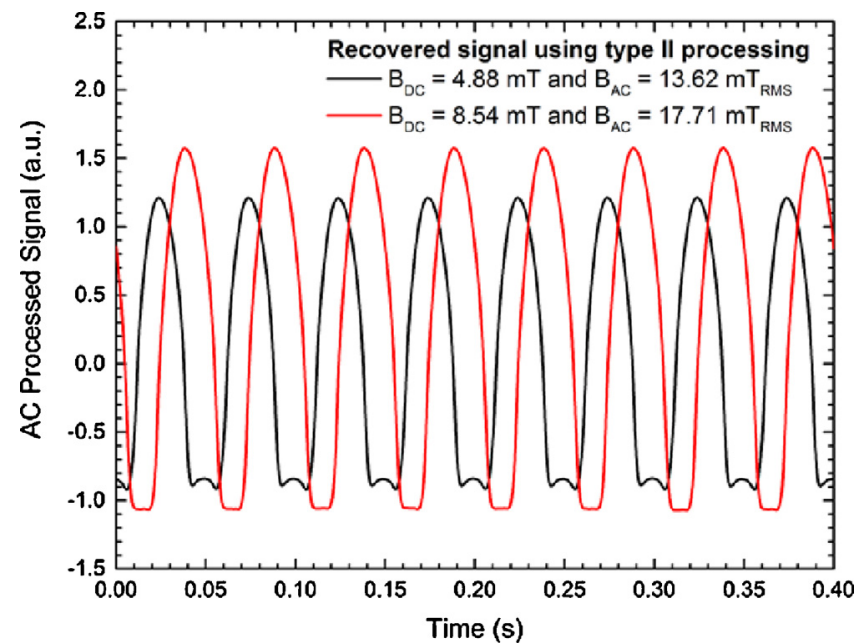

Fig. 8. Type II demodulation AC signals for AC magnetic fields higher than the bias field.

The same measurements where performed simultaneously with the algorithm I, the plots obtained with the two algorithms where indistinguishable and therefore the results have been numerically compared. Considering the results obtained for each independent test maximum errors were calculated and presented in Table 1. The calculation is done considering two times the standard deviation divided by the average $A C$ value in each step for changing magnetic fields higher than $2.25 \mathrm{mT}_{\mathrm{RMS}}$. A maximum error of $1.61 \%$ was found considering a zero magnetic field offset. For other offset values the error is lower than $0.79 \%$ because the sensitivity is higher for low AC magnetic fields. Also, both demodulations algorithms were compared. Although the same response was obtained, the errors were slightly lower using type II algorithm, which makes use of the Arc tangent function. However an isolated case, for a bias of $4.88 \mathrm{mT}$, type II processing gave a slightly superior error. The maximum and minimum improvement obtained with it was 3.02 and $0.1 \%$, respectively. Better resolutions are in general achieved in type II than in type I method because the latter one employs more complex functions such as derivatives and integration, translating in to increased noise.

Fig. 8 shows the signals recovered for two particular cases, where the AC modulation is higher than the bias field. It clearly shows the fundamental frequency of $20 \mathrm{~Hz}$ and a distortion in the lower part of the sinusoidal due to the week response of the magnetostrictive material to low magnetic fields. According to Fig. 5, the region of 0-2 $\mathrm{mT}$ (field going down) and from 0 to $3 \mathrm{mT}$ (field going down) shows very little response. This range is about $5 \mathrm{mT}$ and justifies the flat response of the lower part of both curves.

The signal to Noise ratio was also inspected by the FFT (Fast Fourier Transform) of the recovered demodulated signal as a function of the bias field considering $20 \mathrm{~Hz}$ modulation and bandwidth. In Fig. 9 it is shown the FFT spectrum for a 0 and $4.88 \mathrm{mT}$ bias fields.

The results are also compiled in Fig. 10 for a wider range of bias fields, showing a variation between -63 and $67 \mathrm{~dB}$ for non-zero bias fields with no dependence with the applied bias field. However, the SNR decreases significantly, to $-46 \mathrm{~dB}$ for the $40 \mathrm{~Hz}$ harmonic when no bias field is applied.

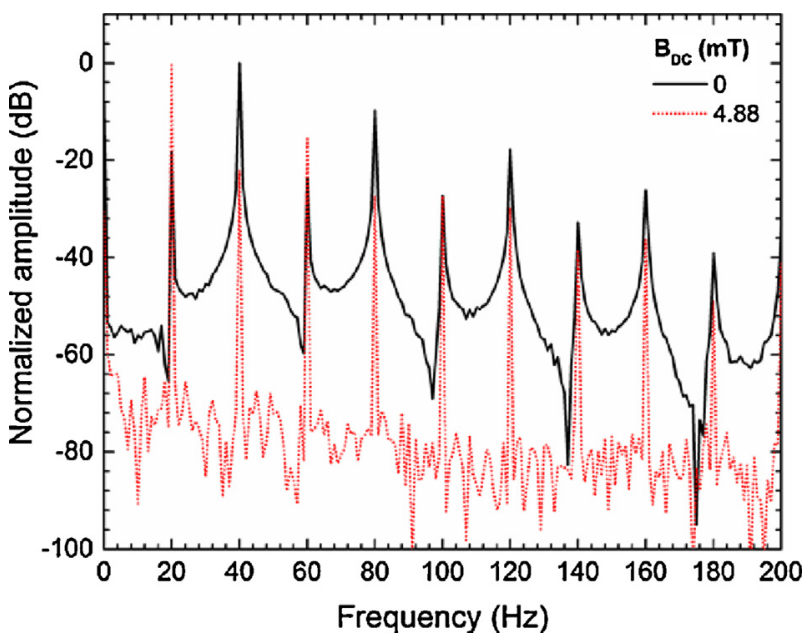

Fig. 9. FFT of the recovered signal when an alternating magnetic field is applied with a bias field of 0 and $4.88 \mathrm{mT}$.

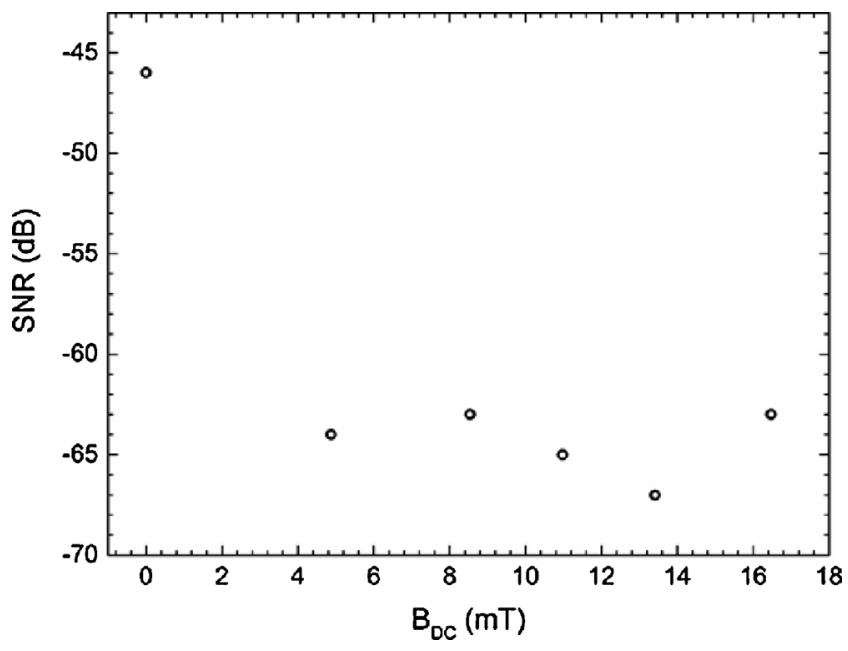

Fig. 10. SNR as a function of the bias field.

In agreement with the DC response of the laser shown in Fig. 5, the best response of the AC current senor is achieved with a low but non-zero biasing field, however the best linearity is obtained for moderate DC fields because the transducer works still far from saturation. These two situations are compared in Fig. 11.

Finally, we have to pint out the foreseeable thermal behavior of this system. This sensor is intended for AC measurements, temperature is a quasi-DC effect because of its variation is slow. Temperature has two effects, the first one is the shift of the wavelength emitted by the laser due to the thermal expansion of the transducer, the type I processing of the acquired signals makes up for this shift so that temperature does not affect the measurement of the AC magnetic field, the type II processing can remove the thermal shift by a DC filter. The second thermal effect is the variation of magnetostrictive strain with temperature; although magnetostrictive curves are very sensitive to stress, it has been demonstrated $[19,20]$ that the response of Terfenol-D is almost temperature inde- 


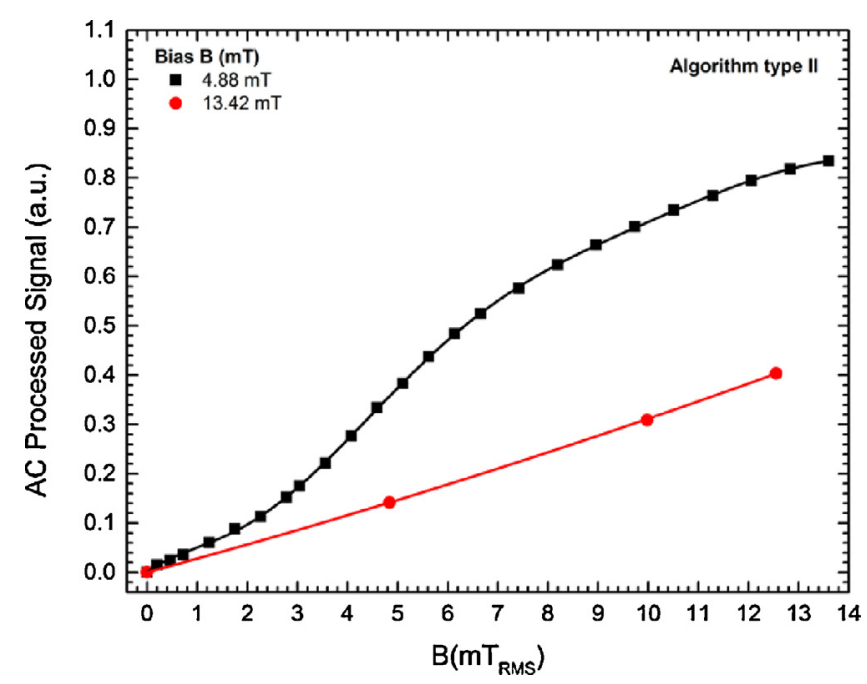

Fig. 11. Type II demodulation algorithm response to AC, using different magnetic field offsets.

pendent in the range of $\left[0^{\circ} \mathrm{C}, 90^{\circ} \mathrm{C}\right]$ for excitation fields between 0 and $40 \mathrm{kA} / \mathrm{m}$. Therefore, it is assumed that the sensor presented here could be reasonably stable within the temperature ranges and magnetic excitations mentioned before. Thermal calibration would be required beyond these ranges and this is a matter of further analysis.

\section{Conclusion}

A magnetic field sensor based on a fiber optic laser has been proposed. The laser emission wavelength is modulated by a magnetostrictive transducer that stretches the Bragg mirrors of the laser. The sensor is interrogated by a Match-Zener interferometer with three outputs at $120^{\circ}$, the three outputs are processed in real time to retrieve the phase variation induced by the AC wavelength shift giving a stable output. Neither thermal stabilization of the gratings nor drift compensation of the interferometer is required. The response of the sensor to AC magnetic fields has been analysed for different strengths of the DC bias filed; the performance of two different demodulation algorithms has been compared. By tuning the DC magnetic field from 0 to $16.47 \mathrm{mT}$ the $\mathrm{AC}$ response showed different responses, where the best sensitivities were achieved for 4.88 and $8.54 \mathrm{mT}$. Additionally, lower sensitivities were observed when a constant magnetic field of $0,13.42$ and $16.47 \mathrm{mT}$ was applied, corresponding to the lower and higher saturation levels of the magnetostrictive material. Moreover, a maximum error of $0.79 \%$ was found for AC magnetic fields above $2.26 \mathrm{mT}_{\mathrm{RMS}}$, with a non-zero bias magnetic field. Furthermore, comparison of both demodulation algorithms revealed the same response, but errors were lower in all tests when using the Arc tangent function based algorithm. The maximum and minimum improvement was $3.02 \%$ and $0.1 \%$, respectively.

\section{Acknowledgements}

This work was supported by project SMARTGRIDS NORTE07-0124-FEDER-000056, financed by the North Portugal Regional Operational Program (ON.2-O Novo Norte), under the National Strategic Reference Framework (NSRF), through the European Regional Development Fund (ERDF), and by national funds, through the Portuguese funding agency, Fundação para a Ciência e a Tecnologia (FCT). Ivo Nascimento would like to acknowledge the financial support of FCT (SFRH/BD/80056/2011). J.L. Cruz and M.V. Andrés acknowledge the financial support of the Ministerio de Economia y Competitividad of Spain, Fondo FEDER and Generalitat Valenciana (projects TEC2013-46643-C2-1-R and PROMETEOII/2014/072).

\section{References}

[1] H. Cao, N. Shi, J. Xu, A novel design of fiber-optic sagnac current sensor, 2012 Fifth Int. Symp. Comput. Intell. Des. (2012) 89-92.

[2] R. Lv, Y. Zhao, D. Wang, Q. Wang, Magnetic fluid-filled optical fiber fabry-pérot sensor for magnetic field measurement, IEEE Photonics Technol. Lett. 26 (2014) 217-219.

[3] G.A. Cranch, G.M.H. Flockhart, C.K. Kirkendall, DFB fiber laser magnetic field sensor based on the lorentz force, Opt. Fiber Sensors (2006) 1-4.

[4] L. Cheng, Z. Guo, J. Han, L. Jin, B. Guan, Ampere force based magnetic field sensor using dual-polarization fiber laser, Opt. Express 21 (2013) 13419-13424.

[5] P. Drexler, P. Fiala, Utilization of faraday mirror in fiber optic current sensors, Radioengineering 17 (2008) 101-107.

[6] M. Takahashi, K. Sasaki, A. Ohno, Y. Hirata, K. Terai, Sagnac interferometer-type fibre-optic current sensor using single-mode fibre down leads, Meas. Sci. Technol. 15 (2004) 1637-1641.

[7] J.L. Cruz, M.V. Andres, M.A. Hernandez, Faraday effect in standard optical fibers: dispersion of the effective Verdet constant, Appl. Opt. 35 (1996) 922-927.

[8] M. Segura, N. Vukovic, N. White, T. May-Smith, W.-H. Loh, F. Poletti, M.N. Zervas, Low birefringence measurement and temperature dependence in metre-long optical fibers, J. Light. Technol. 8724 (2015) 2015.

[9] D.N. Payne, A.B. Barlow, Development of low and high birefringence optical fibers, Quantum Electron. 18 (1982) 477-488.

[10] N. Peng, Y. Huang, S. Wang, T. Wen, W. Liu, Q. Zuo, L. Wang, Fiber optic current sensor based on special spun highly birefringent fiber, IEEE Photonics Technol. Lett. 25 (2013) 1668-1671.

[11] J. Song, P.G. Mclaren, D.J. Thomson, R.L. Middleton, A faraday effect based clamp-on magneto-optical current transducer for power systems, WESCANEX 95 (1995) 329-333.

[12] N. Fisher, D. Jackson, Vibration immunity for a triangular faraday current sensor, Fiber Integr. Opt. 16 (1997) 321-328.

[13] M. Yang, J. Dai, C. Zhou, D. Jiang, Optical fiber magnetic field sensors with TbDyFe magnetostrictive thin films as sensing materials, Opt. Express 17 (2009) 20777-20782.

[14] A. Masoudi, T.P. Newson, Distributed optical fiber dynamic magnetic field sensor based on magnetostriction, Appl. Opt. 53 (2014) 2833-2838.

[15] D.A. Brown, C.B. Cameron, R.M. Keolian, D.L. Gardner, S.L. Garrett, A symmetric $3 \times 3$ coupler based demodulator for fiber optic interferometric sensors, Fiber Opt. Laser Sens. IX 1584 (1991) 328-335.

[16] M.D. Todd, G.A. Johnson, C.C. Chang, Passive light intensity-independent interferometric method for fiber Bragg grating interrogation, Electron. Lett. 35 (1999) 1970-1971

[17] M. Nazarathy, W.V. Sorin, D.M. Baney, S.A. Newton, Spectral analysis of optica mixing measurements, J. Light Technol. 7 (1989) 1083-1096.

[18] D.M. Dagenais, F. Bucholtz, K.P. Koo, A. Dandridge, Detection of low-frequency magnetic signals in a magnetostrictive fiber-optic sensor with suppressed residual signal, J. Light. Technol. 7 (1989) 881-887.

[19] A. E. Clark, High Power Magnetostrictive Transducer Materials-Actuator 92 3rd International Conference on New Actuators, (1992), 127.

[20] E. du, T. de Lacheisserie, D. Gignoux, M. Schlenker, Magnetism: II-materials and applications, magnetostrictive materials, Springer 220 (2002).

\section{Biographies}

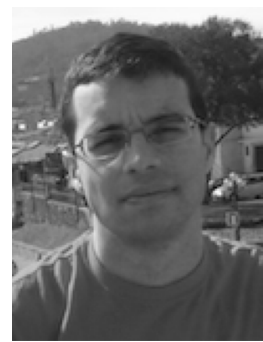

Ivo M. Nascimento was born in Funchal, Portugal, in 1988. He received his B.S. degree in Telecommunications and Electronic Engineer in 2009 and his M.S. degree in Telecommunications and Networks in the University of Madeira, Portugal. Currently he is a Ph.D student in the University of Porto, Portugal and his present research includes fiber optical sensors namely for electric current measurement. 


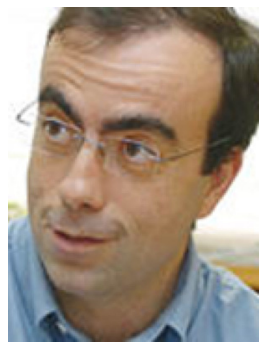

José Manuel Baptista graduated in "Electrical and Computer Engineering" from the University of Porto, Porto, Portugal, in 1991, followed by the M.Sc. degree in "Physics of Laser Communications" from the University of Essex, Colchester, U.K., in 1993, and then he obtained the Ph.D. degree in "Electrical and Computer Engineering" from the University of Porto, in 2002. Currently, he is the ViceRector of the University of Madeira for Research and International Relations. He is Associate Professor of the Exact Sciences and Engineering Department of the University of Madeira, Portugal. He is also Senior Researcher in the Optoelectronics and Electronics Systems Unit at INESC Porto, Portugal. His main scientific areas are the fiber optic sensors, fiber optic communications and fiber optic technologies. He is author of 70 journal publications, 79 communications in national and international conferences, 3 chapter books, 2 patents, reviewer of several scientific publications and member of various international scientific organizations.

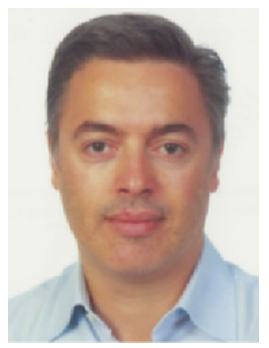

Pedro A.S. Jorge graduated in Applied Physics (Optics and Lasers) at the Univ. of Minho (1996), M.Sc. in Optoelectronics and Lasers at the Physics Department of University of Porto (2000); in 2006 concluded his Ph.D. program at University of Porto in collaboration with the Dept. of Physics and Optical Sciences at the Univ. of Charlotte, North Carolina, USA, with work in luminescence based optical fiber systems for biochemical sensing applications using luminescent nanoparticles. Since 1997 Pedro Jorge has been involved in several research and technology transfer projects related to optical fiber sensing technology, developing new sensing configurations and interrogation techniques for optical sensors. Pedro Jorge is a Senior researcher at INESC Porto where he leads the Biochemical Sensors team exploring the potential of optical fiber and integrated optics technologies in industrial, environmental and medical applications coordinating several projects in these areas. He has more than 150 publications in the fields of sensors in national and international conferences and peer reviewed journals, is author of 3 book chapters and also holds one patent. Pedro Jorge is a member of SPIE.

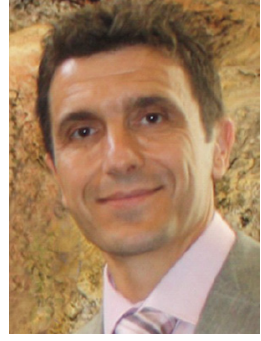

Jose Luis Cruz was born in Cuenca (Spain) in 1964 . He received the Ph.Degree in Physics from the University of Valencia (Spain) in 1992. Initially his career focused on microwave devices for radar applications, afterwards, he joined the Optoelectronics Research Center of the University of Southampton (UK) where he was working in optical fiber fabrication and he is currently Professor of the Applied Physics Department at the University of Valencia where he is conducting research on fiber lasers and amplifiers, photonic crystal fibers, fiber gratings, microwave photonics and sensors. He is co-inventor of six patents related with the above mentioned topics, has published over 110 papers in international journals and more than 150 in conferences. He is OSA member and he regularly acts as reviewer of Elsevier, IEEE and OSA journals, institutions for whom he has reviewed more than 90 manuscripts. He has also been Vice-Dean of the Physics Faculty and Director of the Applied Physics Department for eight years.

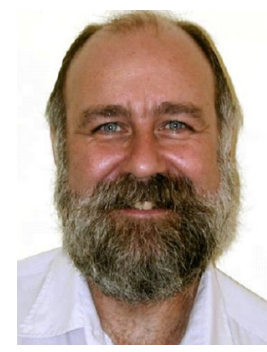

Miguel V. Andrés was born in Valencia (Spain) in 1957 is responsible for the leadership and management of the Group of Fiber Optics at the University of Valencia. He received the B.Sc. and Ph.D. degrees in physics from the University of Valencia, Spain, in 1979 and 1985, respectively. Since 1983, he has successively served as Assistant Professor, Lecturer, and Professor in the Department of Applied Physics, University of Valencia, Valencia, Spain. After a postdoctoral stay (1984-1987) at the Department of Physics, University of Surrey, U.K., he founded the Group of Fiber Optics at the University of Valencia. His current research interests include photonic crystal fibers, in-fiber acousto-optics, fiber lasers and new fiber-based light sources, fiber sensors, microwave photonics, and waveguide theory. 